\title{
Second-Mover Advantage and Price Leadership in Bertrand Duopoly*
}

\author{
Rabah Amir ${ }^{\dagger}$ and Anna Stepanova ${ }^{\ddagger}$
}

This version: Sept 2002

\begin{abstract}
We consider the issue of first versus second-mover advantage in differentiated-product Bertrand duopoly with general demand and asymmetric linear costs. We generalize existing results for the cases where prices are either strategic substitutes and/or complements, dispensing with common extraneous assumptions. We show that a firm with a sufficiently cost lead over its rival has a first mover advantage. For the linear version of the model, we invoke a natural endogenous timing scheme coupled with equilibrium selection according to riskdominance. This yields sequential play with the low-cost firm as leader as the unique equilibrium outcome.
\end{abstract}

Key words and phrases: Price competition, endogenous timing, first/second-mover advantage, risk dominance.

JEL codes: L 13, C 72.

*The authors are grateful to Isabel Grilo, Sjaak Hurkens and Jean-Francois Mertens for helpful conversations on the subject of this paper, and to two anonymous referees of this journal for their suggestions. R. Amir also gratefully acknowledges the support of the Center for Industrial Economics at the University of Copenhagen, where he was when most of the work presented here was conducted.

${ }^{\dagger}$ CORE and Department of Economics, Universite Catholique de Louvain, 1348 Louvain-la-neuve, Belgium (Corresponding author: amir@core.ucl.ac.be)

${ }^{\ddagger}$ Department of Economics, University of Southern Denmark at Odense, 5230 Odense M, Denmark. (E-mail: avs@sam.sdu.dk) 


\section{Introduction}

The classical issue in traditional oligopoly theory dealing with the appropriateness of the equilibrium concept, Cournot-Nash or Stackelberg, in various imperfectly competitive settings has enjoyed a major revival over the last fifteen years. This revival consists of two main strands of research with some overlap. The first deals with the determination of first- and second-mover advantages in given subclasses of the general class of duopoly games characterized by monotone best-responses (upward or downward-sloping), and monotone profits in rival's actions. In other words, this strand compares the equilibrium payoffs of the two firms in the two sequential games of perfect information obtained by considering both orders of moves. This strand includes ${ }^{1}$ Gal-Or (1985) and Dowrick (1986).

The second strand of research deals with the issue of endogenous timing. Its guiding premise is that in duopoly models the determination of simultaneity versus sequentiality of moves, as well as of the assignment of roles to the players in the latter case, should be completely endogenous. In other words, the order of play in a given two-player game ought to reflect the players' own intrinsic incentives, in the absence of any natural exogenously determined timing structure, Hamilton and Slutsky (1990) and Amir (1995). ${ }^{2}$

The present paper relates to both strands and deals with duopoly price competition with differentiated products and constant unit costs. ${ }^{3}$ It is widely believed that price competition is typically characterized by a second-mover advantage. As Bertrand's classical critique of Cournot shows, this intuition certainly holds in an extreme form in the case of homogeneous products, owing to the totally discontinuous nature of each firm's demand along the price diagonal. Furthermore, this intuition has also been strongly reinforced as a second-mover advantage also prevails in the differentiated-products case when firms are identical.

The present paper has two related objectives. Firstly, we generalize the well-known

1 Mailath (1993) and Daughety and Reinganum (1994), among others, have signalling frameworks.

2 Studies with specific oligopolistic settings are numerous; they include Boyer and Moreaux (1987), Robson (1990), Deneckere and Kovenock (1992), Leininger (1993), Anderson and Engers (1992), van Damme and Hurkens (1998, 1999), Pastine and Pastine (1999), Amir and Grilo (1999), and Amir, Grilo and Jin (1999), amomg others. Studies dealing with abstract game formulations include d'Aspremont and Gerard-Varet (1980), Rosenthal (1991) and van Damme and Hurkens (1996).

3 Price leadership in the form of a dominant firm has been an extensively investigated topic by early oligopoly theorists, see e.g. Stigler (1947) and Markham (1951). 
results in the literature on first/second mover advantage in price competition with constant unit costs, by removing the common (sometimes tacit) assumptions of concavity of profits in own action (or continuity and single-valuedness of the reaction curves), and of existence and uniqueness of the Bertrand equilibrium. To do so, we invoke the recent results of supermodular optimization/games. ${ }^{4}$ In the process, we also clarify the crucial role played by the strategic complementarity or substitutability of prices in determining timing advantage in price competition with asymmetric firms. To this end, we consider all three possible cases that can arise, making minimal assumptions on primitives leading to each case. We prove that when both optimal reactions slope upwards, at least one firm has a second-mover advantage. When both optimal reactions slope downwards, both firms have a first-mover advantage. In the mixed case, the firm with a downward-sloping reaction always has a firstmover advantage. The supermodularity approach allows for clear insights and interpretations as it does not rely on superfluous assumptions (such as concavity of profits) that would be common across all three cases. The same minimal assumptions on demand allow a sharp determination of timing advantage while at the same time taking care of all the critical attending issues, such as existence and possible multiplicity of equilibrium.

We also investigate the scope of the second-mover advantage under strategic complementarity of prices, asymmetric firms and linear demand for differentiated products. ${ }^{5}$ We show that when the unit costs of the two firms are sufficiently different (close), the low-cost firm has a first-mover (second-mover) advantage. On the other hand, the high-cost firm always has a second-mover advantage, thus partly confirming the conventional intuition.

The second objective is to consider the issue of endogenous timing of moves for the linear price duopoly with asymmetric unit costs, according to the concept proposed by Hamilton and Slutsky (1990) in their extended game with observable delay. While it is well-known that sequential play with both orders of move are the only two pure-strategy subgameperfect equilibria of the extended game for the model at hand, no compelling argument has been advanced to select one equilibrium, thereby providing an explanation for a natural 4 See Topkis (1978), Vives (1990), and Milgrom and Roberts (1990), among others.

5 This restriction is due to reasons of tractability. The analysis of the rest of the first part of the paper and of the closely related second part (in particular the issue of risk dominance) requires a precise comparison of equilibrium payoffs that unfortunately does not seem possible under full generality. 
(endogenous) price leader. To this end, we invoke the equilibrium selection concept of riskdominance of Harsanyi and Selten (1988). Applied to coordination games with two purestrategy strict equilibria, this procedure picks the equilibrium that has the largest basin of attraction in the initial beliefs players ascribe to each other's behavior. In other words, it minimizes the risk of a coordination failure due to strategic uncertainty ${ }^{6}$.

In the context at hand, risk-dominance always selects the sequential play outcome where the more efficient (or low unit cost) firm acts as leader. The same outcome is also Paretodominant over the other equilibrium when the unit costs are sufficiently different from one another, while the two equilibrium outcomes cannot be Pareto ranked otherwise.

We conduct a profit comparison between the leader and the follower at the selected equilibrium. The leader (low-cost firm) makes a higher profit only if the unit costs are sufficiently different. Thus, a benefit to moving second is still at work here, as the follower can end up with higher profit despite his cost disadvantage, if sufficiently small. In selecting between the two sequential play outcomes, risk dominance favors the high-cost (or small size) firm by assigning it the more favorable second-mover status ${ }^{7}$.

In conclusion then, combining Hamilton and Slutsky's extended game with observable delay and risk dominance allows for a simple and natural explanation for the endogenous emergence of price leadership, with the leader being the strong firm, as originally envisaged by Stackelberg who was well aware though that he had put forward no compelling reason to justify his assignment of roles. Analysing the same price game but relying on the second endogenous timing scheme - with action commitment - of Hamilton and Slutsky (1990) and risk-dominance as a selection concept, van Damme and Hurkens (1998) show that the same sequential outcome prevails. While the economic interpretation and motivation of the two results are the same, the attending analysis is quite different, due to the a priori unrelated timing schemes used. ${ }^{8}$ Furthermore, there is no a priori reason at all to expect the two

6 In coordination games, risk-dominance emerges as a powerful selection concept in experimental settings (Van Huyck et al, 1990, and Cooper et al, 1990), and in evolutionary games (Kandori et al, 1993 and Ellison, 1993). The risk-dominant equilibrium is often selected even when it is dominated by another equilibrium. 7 Interestingly, using a model of price competition where firm capacities play a key role, Kovenock and Deneckere (1992) come to an analogous intuitive explanation for the emergence of the large firm as leader. 8 In both the present paper and in van Damme and Hurkens (1998, 1999), risk dominance is applied to the reduced game obtained by replacing the subgames following the announcement stage of the extended game by their unique equilibrium payoffs, in accordance with subgame perfection. This methodological point will 
timing schemes to yield the same outcome. ${ }^{9}$ Thus the two results are natural complements in establishing the robustness of the common conclusion to the timing scheme.

Besides its theoretical appeal, the literature on endogenous timing can also yield some direct policy-relevant insights. A good understanding of the timing incentives facing firms under noncooperative behavior would be a pre-requisite to a more general definition of market structure or industry concentration, and a delimitation criterion for collusive behavior, in antitrust policy. Indeed, in a duopoly where a sequential-move outcome yields payoffs that Pareto dominate the simultaneous-move outcome, it is reasonable to consider the former, rather than the latter, as the more plausible outcome of a static-like model of the industry. While relative to the simultaneous-move equilbrium, the sequential-move outcome will then yield profits that go part of the way towards a collusive outcome, no collusion need actually occur. These considerations should clearly be integrated into antitrust policy formulation, with proper empirical testing developed. Related issues are discussed in Daughety (1990).

While the literature on endogenous timing of moves is typically silent on how a sequential outcome in duopoly might emerge in a practical sense, one could think of some real-life practices that may well lead to a sequential-like outcome in duopoly competition. One of these is the most-favored customer pricing policy, whereby a firm commits to reimburse consumers the price differential if it should lower prices in the future. In a simple two-stage model, Cooper (1986) shows that this allows duopoly firms to coordinate on a Stackelberglike outcome, if one of them offers such a pricing guarantee. The latter firm ends up as a Stackelberg leader in the pricing game. Other pricing practices with similar effects on competition are discussed in Salop (1986).

The paper is organized as follows. Section 2 describes the general model, the equilibrium concepts, and the comparative results for first and second movers. Section 3 shows that these results cannot be strengthened and deals with the issue of endogenous timing, in the specific context of linear demand. Section 4 has all the proofs. An appendix, with a brief

be discussed further at the end of Section 3 .

9 In fact, for the linear homogeneous Cournot duopoly, using action commitment, van Damme and Hurkens (1999) obtain sequential play with the low-cost firm as leader again, while Amir and Grilo (1999), using observable delay, obtain simultaneous moves. Under restrictive conditions on demand, Amir and Grilo (1999) do obtain a sequential outcome, but then with the high-cost firm as leader! 
and simple outline of the lattice-theoretic notions and results used here, is given.

\section{Model and Results}

Consider the standard model of duopolistic price competition with differentiated goods. Firm $i$ charges price $p_{i}$, faces demand $D_{i}\left(p_{1}, p_{2}\right)$ and is assumed to have linear production costs with marginal cost $c_{i}, i=1,2$. The profit of firm $i$ is then given by

$$
\Pi_{i}\left(p_{1}, p_{2}\right)=\left(p_{i}-c_{i}\right) D_{i}\left(p_{1}, p_{2}\right)
$$

We consider three different games of price competition that are distinguished only by their timing structure: A simultaneous-move game $G$ and two games with sequential moves and perfect information, $G_{1}$ and $G_{2}$. A pure strategy for firm $i$ in game $G$ is to choose an element of its price set $P_{i} \triangleq\left[c_{i}, \bar{p}_{i}\right]$, where the upper bound $\bar{p}_{i}$ can be justified in many cases, e.g. via an argument of bounded income. In game $G_{i}$, firm $i$ (the leader) moves first, choosing a pure strategy $p_{i} \in P_{i}$, and the other firm (the follower) moves after observing $p_{i}$, choosing its pure strategy $\gamma\left(p_{i}\right)$, where $\gamma(\cdot)$ is a mapping from $P_{i}$ to $P_{j}, j \neq i$.

For each of these games, let us define the associated equilibrium concept. A pair $\left(p_{1}^{*}, p_{2}^{*}\right)$ constitutes a Nash (or Bertrand) equilibrium in game $G$ if for all $p_{1} \in P_{1}, p_{2} \in P_{2}$

$$
\Pi_{1}\left(p_{1}^{*}, p_{2}^{*}\right) \geq \Pi_{1}\left(p_{1}, p_{2}^{*}\right) \text { and } \Pi_{2}\left(p_{1}^{*}, p_{2}^{*}\right) \geq \Pi_{2}\left(p_{1}^{*}, p_{2}\right)
$$

For games $G_{1}$ and $G_{2}$, the equilibrium concept is subgame-perfect equilibrium or SPE (also known as Stackelberg equilibrium in traditional oligopoly theory ${ }^{10}$ ), which is defined as follows, say for game $G_{1}$. A pair $\left(p_{1}^{*}, \gamma^{*}(\cdot)\right)$ is a SPE for game $G_{1}$ if for all $p_{1} \in P_{1}, p_{2} \in P_{2}$,

$$
\Pi_{2}\left(p_{1}, \gamma^{*}\left(p_{1}\right)\right) \geq \Pi_{2}\left(p_{1}, p_{2}\right) \text { and } \Pi_{1}\left(p_{1}^{*}, \gamma^{*}\left(p_{1}^{*}\right)\right) \geq \Pi_{1}\left(p_{1}, \gamma^{*}\left(p_{1}\right)\right)
$$

In other words, a SPE imposes the following restrictions on players' behavior:

(i) the second-mover must be using as strategy a (single-valued) selection from his bestresponse correspondence, defined as usual by $r_{2}\left(p_{1}\right) \triangleq \arg \max \left\{\Pi_{2}\left(p_{1}, p_{2}\right): p_{2} \in P_{2}\right\}$, and

10Stackelberg's solution concept is not well-defined for one-shot games. It corresponds precisely to the notion of SPE of a two-stage game with sequential moves, perfect information and exogenously given first and second movers: See [Friedman, 1977, p. 78] for an early insightful discussion of this issue. 
(ii) the first-mover must choose a price that maximizes his payoff given the anticipation of a rational reaction (according to the strategy $\gamma^{*}(\cdot)$ ) by the rival.

The following standard assumption is made throughout the paper (for $i=1,2$ ):

(A1) The demand function $D_{i}\left(p_{1}, p_{2}\right)$ is twice continuously differentiable when $>0$ and

$$
\partial D_{i}\left(p_{1}, p_{2}\right) / \partial p_{i}<0 \quad \text { and } \quad \partial D_{i}\left(p_{1}, p_{2}\right) / \partial p_{j}>0 \text { for all }\left(p_{1}, p_{2}\right) \in P_{1} \times P_{2} \text {. }
$$

The first inequality says that each demand is downward sloping in own price, and the second that goods are substitutes (each demand increases with the price of the other good.)

Gal-Or (1985) shows that in case of symmetric firms, there is a second-mover (first-mover) advantage for both players when each profit function is strictly concave in own action and strictly increasing (decreasing) in rival's action, and reaction curves are upward (downward) sloping. Dowrick (1986) derives this result for asymmetric duopoly.

Before formulating our results, let us extend the definition of the notions of "first- and second-mover advantage" to asymmetric games. We say that firm $i$ has a first (second) mover advantage if its equilibrium payoff in $G_{i}\left(G_{j}\right)$ is higher than in $G_{j}\left(G_{i}\right)$.

Our minimal assumptions for determining timing advantage below will also include provisions for the relevant equilibria of our games to exist. While game $G$ may have multiple Bertrand-Nash equilibria, the games $G_{1}$ and $G_{2}$ will (essentially) not have multiple SPEs, as we now argue. Multiple SPEs for (say) game $G_{1}$ can arise in two different ways. The first is that, given the follower's strategy $\gamma^{*}$, the leader's payoff, $\Pi_{1}\left(p_{1}, \gamma^{*}\left(p_{1}\right)\right)$, may have more than one argmax. While possible, this situation is generically removable, in that a perturbation of the game parameters or primitives will result in a unique argmax.

The second source of nonuniqueness of SPEs is that at the leader's optimal choice $p_{1}^{*}, r_{2}(\cdot)$ is multi-valued. In this case, Amir et al (1999) show that there is a unique SPE, $\left(p_{1}^{*}, \bar{r}_{2}(\cdot)\right)$, where $\bar{r}_{2}$ is the maximal selection of $r_{2}$, due to the fact that the goods are substitutes or that each profit is increasing in rival's price ${ }^{11}$. In view of these arguments, we henceforth assume uniqueness of the SPE of games $G_{1}$ and $G_{2}$, but not of the game $G$.

11The proof of this fact is based upon the fact that at any other candidate SPE where the follower reacts to $p_{1}^{*}$ by any action in $r_{2}\left(p_{1}^{*}\right)$ other than $\bar{r}_{2}\left(p_{1}^{*}\right)$, the leader will have a profitable deviation to $p_{1}^{*}+\varepsilon$, for some small $\varepsilon>0$ such that $r_{2}$ is single-valued at $p_{1}^{*}+\varepsilon$. This argument relies crucially on the monotonicity of the best responses and of each payoff, in the other player's strategy. 
Our first proposition requires the following assumption on the demand functions:

(A2) $D_{i}\left(p_{1}, p_{2}\right)$ is strictly log-supermodular on $P_{1} \times P_{2}, i=1,2$.

This assumption implies that

$$
\Delta_{D_{i}} \triangleq \frac{\partial^{2} \log D_{i}\left(p_{1}, p_{2}\right)}{\partial p_{1} \partial p_{2}}=D_{i}\left(p_{1}, p_{2}\right) \frac{\partial^{2} D_{i}\left(p_{1}, p_{2}\right)}{\partial p_{1} \partial p_{2}}-\frac{\partial D_{i}\left(p_{1}, p_{2}\right)}{\partial p_{i}} \frac{\partial D_{i}\left(p_{1}, p_{2}\right)}{\partial p_{j}} \geq 0
$$

and, conversely, the strict version of this inequality implies (A2). The main implication of (A2) is that it leads to reaction correspondences that are nondecreasing (in the sense that each selection is nondecreasing) but need not be single-valued or continuous ${ }^{12}$. (A2) has a very appealing and precise interpretation: The price elasticity of firm $i$ 's demand increases in the rival's price, Milgrom and Roberts (1990). This is a very intuitive and general condition, though clearly not a universal one. It is satisfied in particular if $\partial^{2} D_{i}\left(p_{1}, p_{2}\right) / \partial p_{1} \partial p_{2} \geq 0$, i.e. if a higher price by a firm's rival does not lower the responsiveness of the firm's demand to a change in own price. See [Milgrom and Roberts, 1990 and Vives, 2000, pp. 151-154] for a list of examples that includes most widely used specifications (such as linear demand), and a more detailed discussion of the scope of this assumption.

Proposition 1 Under Assumptions (A1) and (A2), at least one of the firms has a secondmover advantage.

The issue of moving advantage is not conclusive for the other firm under our general assumptions. This point is investigated in detail below for the special case of linear demand for differentiated products, for which it is shown that both first and second-mover advantages are possible for the other firm, depending on the unit cost difference.

This Proposition is closely related to a result of Dowrick (1986). Our proof deals explicitly and rigorously with the possible discontinuity and multi-valuedness of the optimal reactions (or lack of any concavity-type assumptions on profits), as well as with existence and possible nonuniqueness of the relevant equilibrium points of $G, G_{1}$ and $G_{2}$. In addition, this is all done with minimal interpretable assumptions placed directly on primitives.

Another result relating the same three games has appeared in the literature under some extra assumptions beyond $(A 1)$ and $(A 2)$ : See Dowrick (1986) and Hamilton and Slutsky 12An alternative sufficient condition is the supermodularity of the profit function $\Pi_{i}$ in the prices (Vives, 1990), i.e. $\partial D_{i}\left(p_{1}, p_{2}\right) / \partial p_{j}+\left(p_{i}-c_{i}\right) \partial^{2} D_{i}\left(p_{1}, p_{2}\right) / \partial p_{1} \partial p_{2} \geq 0$. 
(1990). It shows that each firm prefers both games $G_{1}$ and $G_{2}$ to game $G$, but does not compare games $G_{1}$ and $G_{2}$ to each other. In other words, each firm prefers to be a Stackelberg player (whether leader or follower) to playing simultaneously. Amir, Grilo and Jin (1999) derive the same result in the present (more general) setting ${ }^{13}$.

The next result deals with the case where the reaction correspondences of both firms are downward- sloping. This requires the following assumption on demand functions:

(A3) $D_{i}\left(p_{1}, p_{2}\right)$ is strictly log-submodular in $P_{1} \times P_{2}, i=1,2$.

This assumption implies that

$$
\Delta_{D_{i}}=D_{i}\left(p_{1}, p_{2}\right) \frac{\partial^{2} D_{i}\left(p_{1}, p_{2}\right)}{\partial p_{1} \partial p_{2}}-\frac{\partial D_{i}\left(p_{1}, p_{2}\right)}{\partial p_{i}} \frac{\partial D_{i}\left(p_{1}, p_{2}\right)}{\partial p_{j}} \leq 0
$$

and, conversely, the strict version of this inequality implies (A3). The main implication of (A3) is that reaction correspondences are nonincreasing (in the sense that each selection is nonincreasing). (A3) can be interpreted as follows: The price elasticity of firm $i$ 's demand decreases in the rival's price. This is a rather counterintuitive and restrictive assumption, but a robust one nonetheless. It clearly requires that $\partial^{2} D_{i}\left(p_{1}, p_{2}\right) / \partial p_{1} \partial p_{2}$ be strongly negative. An example of such a demand is given below.

Proposition 2 Under Assumptions (A1) and (A3), each firm has a first mover advantage.

This result holds that with decreasing best-responses, price competition is characterized by the same timing advantage as quantity competition: Both firms prefer to lead than to follow. Thus the fact that a firm's profit as a function of rival's action is increasing in price competition and decreasing in quantity competition does not play a role in this comparison.

The next result deals with the mixed case when one of the firms (say firm 2) has a logsupermodular demand function, and hence an upward-sloping reaction curve, and firm 1 has a log-submodular demand function, hence a downward-sloping reaction curve. Quasi-concavity

$\overline{13[\text { Amir, Grilo and Jin (1999), Lemma 4.1] }}$ proves the part that each firm prefers being a Stackelberg leader than a simultaneous player for Bertrand competition precisely under our assumptions here, while Amir and Grilo (1999) provides the analogous result for Cournot competition. It is worthwhile to point out that this fact requires serious proof and is not necessarily true without the underlying structure (i.e. the monotonicity of the best-responses and of each profit in rival's action, and the continuum nature of the action sets). This fact is always true though if the best-responses are single-valued continuous functions. 
is needed only to guarantee existence of a Bertrand equilibrium (as Tarski's theorem clearly does not apply when the two optimal reactions are monotone in different directions). An alternative to making this assumption is simply to assume existence directly. ${ }^{14}$

Proposition 3 Let Assumption (A1) and the quasi-concavity of each firm's profit function in own price hold for both firms, $D_{2}$ be strictly log-supermodular, and $D_{1}$ be strictly logsubmodular. Then firm 1 has a first mover advantage.

Here, the issue of moving advantage is not conclusive for firm 2 under the given assumptions. While unusual, this situation is only restrictive in that firm 1's reaction curve is decreasing in $p_{2}$, as in the previous result. In particular, the two products can well be substitutes in demand, according to Assumption (A1), while simultaneously having a mixed strategic complement-substitute relationship. Here is a robust example.

Example. Consider the following system of demand functions:

$$
D_{1}\left(p_{1}, p_{2}\right)=\frac{1}{\left(p_{1}+1\right)^{2}}+\left(p_{2}+1\right) e^{-p_{1}} \text { and } D_{2}\left(p_{1}, p_{2}\right)=a-p_{2}-e^{-p_{1}}, a>0 .
$$

$D_{1}$ satisfies Assumption (A1) since $\frac{\delta D_{1}\left(p_{1}, p_{2}\right)}{\delta p_{1}}=\frac{-2}{\left(p_{1}+1\right)^{3}}-\left(p_{2}+1\right) e^{-p_{1}}<0, \frac{\delta D_{1}\left(p_{1}, p_{2}\right)}{\delta p_{2}}=e^{-p_{1}}>0$. $D_{1}$ is strictly log-submodular (Assumption A3) as

$$
\Delta_{D_{1}}=D_{1}\left(p_{1}, p_{2}\right) \frac{\partial^{2} D_{1}\left(p_{1}, p_{2}\right)}{\partial p_{1} \partial p_{2}}-\frac{\partial D_{1}\left(p_{1}, p_{2}\right)}{\partial p_{1}} \frac{\partial D_{1}\left(p_{1}, p_{2}\right)}{\partial p_{2}}=\frac{-p_{1} e^{-p_{1}}}{\left(p_{1}+1\right)^{3}}<0 .
$$

Likewise, $D_{2}$ satisfies (A1) since $\frac{\delta D_{2}\left(p_{1}, p_{2}\right)}{\delta p_{2}}=-1, \frac{\delta D_{2}\left(p_{1}, p_{2}\right)}{\delta p_{1}}=e^{-p_{1}}>0$, and is strictly logsupermodular (Assumption A2) since $\Delta_{D_{2}}=e^{-p_{1}}>0$.

It can be verified that both demand functions lead to quasi-concave profits in own price. ${ }^{15}$ Thus, this example fits Proposition 3, with firm 1 having the first mover advantage.

At an intuitive level, one generally thinks of price competition as characterized by a second-mover advantage. Indeed, the option to undercut the rival's price often seems appealing. With homogeneous products, price undercutting allows a firm to capture the entire 14A condition on primitives that ensures quasi-concavity of firm i's profit is (Caplin and Nalebuff, 1991): $1 / D_{i}$ is convex in $p_{i}$, i.e., $D_{i}\left(p_{1}, p_{2}\right) \frac{\partial^{2} D_{i}\left(p_{1}, p_{2}\right)}{\partial p_{i}^{2}}-2\left(\frac{\partial D_{i}\left(p_{1}, p_{2}\right)}{\partial p_{i}}\right)^{2} \leq 0$. This condition is, in general, not comparable to (A2)-(A3).

15 Also, as the Jacobian of the demand system $\left(D_{1}, D_{2}\right)$ is negative definite and $\frac{\delta D_{1}\left(p_{1}, p_{2}\right)}{\delta p_{2}}=\frac{\delta D_{2}\left(p_{1}, p_{2}\right)}{\delta p_{1}}=e^{-p_{1}}$, this demand system can be derived from a representative consumer maximizing a concave utility function. 
market, and thus conveys an extreme advantage. With differentiated products and identical firms, a second-mover advantage always prevails when prices are strategic complements. An important message so far is that the strategic complementarity of prices is crucially needed for a second-mover advantage to prevail, even under symmetry (Propositions 2-3.)

Given strategic complementarity of prices, it is then natural to ask whether a secondmover advantage would survive high degrees of firm asymmetry and of product differentiation. Besides its independent interest, this will also be used in investigating the issue of endogenous timing for the price game, as will become clear later. To this end, the case of linear demand and costs provides a convenient framework of analysis, in that it does not seem possible to answer the issues at hand under the full generality used so far. For the first issue, the question is settled in the negative, even in the linear specification: The low-cost firm enjoys a first-mover advantage if its efficiency edge over its rival is substantial enough.

\section{Scope of Second-Mover Advantage and Endogenous Timing}

This section deals with two related issues, in the special but commonly used framework of symmetric linear demand and costs. The first issue is whether a second-mover advantage survives high levels of firm asymmetry, and the second is endogenous timing as proposed by Hamilton and Slutsky's extended game with observable delay. While it is well-known that sequential play with both orders of play emerge as the pure equilibrium outcomes, the selection of a natural leader will require additional arguments of risk dominance and Pareto dominance. Since the latter entails a comparison of the firms' SPE payoffs under both orders of move, it amounts to the same thing as the first issue above (scope of second mover advantage, cf. Lemma 5 below.)

The symmetric linear demand system is w.l.o.g. given by

$$
D_{i}\left(p_{1}, p_{2}\right)=\max \left\{a-p_{i}+b p_{j}, 0\right\}, \text { where } 0<b<1,
$$

with the profit of firm $i$ still given by (1). Furthermore, w.l.o.g., we assume that $c_{1}>c_{2}$.

To guarantee interiority of solutions for all three games at hand, the following assumption on demand and unit costs is needed. It guarantees that quantities are positive and prices are above marginal costs at the unique equilibrium of each game: 
(B) The demand function (2) satisfies: $a>M_{1}$, where $M_{1} \triangleq \frac{c_{1}\left(2-b^{2}\right)-b c_{2}}{2+b}$.

Lemma 4 Under Assumption (B) and linear demand and cost functions, the unique BertrandNash equilibrium for game $G$ is given by $(i, j=1,2, i \neq j)$ :

$$
p_{i}^{N}=\frac{a(2+b)+b c_{j}+2 c_{i}}{4-b^{2}} .
$$

The Stackelberg equilibrium prices and profits of the game $G_{i}$ are (here ${ }^{16} \quad i, j=1,2, i \neq j$ ):

$$
\begin{gathered}
p_{i}^{L}=\frac{a(2+b)+b c_{j}}{2\left(2-b^{2}\right)}+\frac{c_{i}}{2} \text { and } p_{j}^{F}=\frac{\left(4-b^{2}\right)\left(a+c_{j}\right)+2 a b}{4\left(2-b^{2}\right)}+\frac{c_{i} b}{4} \\
\Pi_{i}^{L}=\frac{\left[a(2+b)-\left(2-b^{2}\right) c_{i}+b c_{j}\right]^{2}}{8\left(2-b^{2}\right)} \text { and } \Pi_{j}^{F}=\frac{\left[a\left(4+2 b-b^{2}\right)+b\left(2-b^{2}\right) c_{i}+\left(3 b^{2}-4\right) c_{j}\right]^{2}}{16\left(2-b^{2}\right)^{2}}
\end{gathered}
$$

\subsection{Scope of Second-Mover Advantage}

We derive a characterization of the scope of validity of the second-mover advantage in terms of the level of demand $a$ or the units costs. It is shown that it always holds for the high-cost firm, and extends to the low-cost-firm if and only if the unit costs are close. Let

$$
M_{2} \triangleq \frac{\left(2-b^{2}\right) c_{1}-\left(2 b^{2}+b-2\right) c_{2}+\sqrt{2\left(2-b^{2}\right)}(1+b)\left(c_{1}-c_{2}\right)}{4+3 b} .
$$

As will be seen in the Appendix, $M_{2}$ is the unique feasible value of $a$ for which firm 2 is indifferent between leading and following, i.e., $a=M_{2} \Leftrightarrow \Pi_{2}^{L}=\Pi_{2}^{F}$.

The next result compares the SPE payoffs of the two-stage games $G_{1}$ and $G_{2}$, and thus also constitutes the Pareto dominance argument to be used in Proposition 6 below.

Lemma 5 Under linear demand and cost functions, and Assumption (B),

(i) firm 1 (high-cost firm) has a second-mover advantage $\left(\Pi_{1}^{F} \geq \Pi_{1}^{L}\right), \forall a$.

(ii) firm 2 (low-cost firm) has a first-mover advantage $\left(\Pi_{2}^{L} \geq \Pi_{2}^{F}\right)$ when a $\in\left[M_{1}, M_{2}\right]$, but a second-mover advantage $\left(\Pi_{2}^{F} \geq \Pi_{2}^{L}\right)$ when $a \in\left[M_{2}, \infty\right)$, where $M_{1}$ is given in assumption $(B)$ and $M_{2}$ in (5). Furthermore, the scope for firm 2's first-mover advantage, $M_{2}-M_{1}$, increases with the cost difference ${ }^{17}\left(c_{1}-c_{2}\right)$.

$\overline{16}$ The superscripts $L$ and F stand for Stackelberg leader and follower, respectively. Thus $p_{i}^{L}$ and $p_{i}^{F}$ are firm $i$ 's prices in $G_{i}$ and $G_{j}$, respectively. This notation is meant to avoid (confusing) superscripts for games.

${ }_{17}$ In fact, this scope shrinks with $c_{1}-c_{2}$ since $M_{2}-M_{1}=\frac{(1+b)\left(2 b^{2}-4+(2+b) \sqrt{\left.4-2 b^{2}\right)}\right.}{(2+b)(4+3 b)}\left(c_{1}-c_{2}\right)$, see Appendix. 
This establishes that the conjecture that a second-mover advantage always exists in Bertrand competition is generally false. It fails to hold globally (i.e. for all feasible parameter values) even in the most common specification: linear demand and constant unit costs. For a clear interpretation of Lemma 5 , note that the condition $a \in\left[M_{1}, M_{2}\right]\left(\right.$ resp. $\left.a \in\left[M_{2}, \infty\right)\right)$ can be restated in terms of unit costs being sufficiently different (close), i.e. in terms of $c_{1}-c_{2}$ being large (small) enough ${ }^{18}$. Thus, the well-known second-mover advantage in price competition relies crucially on near-symmetry between the two firms in the differentiatedproducts case, or on the discontinuity of the single firms' demand functions along the price diagonal inherent in the homogeneous-product case.

\subsection{Endogenous timing}

The issue of endogenous timing for the linear Bertrand duopoly is examined according to the extended game with observable delay of Hamilton and Slutsky (1990). They show that for the model at hand the endogenous outcome is sequential play with both leader-follower configurations. Restricting attention to SPE, the analysis reduces to a $2 \times 2$ reduced game of coordination, when the four possible subgames are replaced by their unique equilibrium payoffs. In view of the fact that this $2 \times 2$ reduced game is then one of coordination, it is natural to invoke equilibrium selection arguments according to the criteria of risk and Pareto dominance. Sequential play with the low-cost firm as leader emerges as the unique outcome. Some methodological aspects of the use of risk dominance are discussed later.

The extended game is defined as follows: at the first or preplay stage to the "basic game" 19 , players simultaneously decide whether to move early or late in the "basic game", independently of each other. Players are committed to this choice. After preplay stage is realized, the timing choice of each player is announced. The second stage (or "basic game") is then played according to these timing decisions: with simultaneous play if both players decide to move at the same time (whether early or late), and with sequential play under

18While the interpretation via unit costs is much more intuitive, an exact statement of the conditions is much more cumbersome than via the level of demand $a$, as given. The computational details are left out. ${ }^{19}$ The "basic game" actually stands for three different games: $G, G_{1}$ and $G_{2}$, so it is defined up to the timimg structure. With this clarified, we continue to abuse terminology by referring to all 3 as the "basic game". 
perfect information otherwise (with the order of moves as announced by the players). ${ }^{20}$ Thus, the SPE of this extended game induce an endogenous sequencing of moves in the "basic game". In view of the uniqueness of equilibrium for each of the subgames of the extended game (which are given by the games $G, G_{1}$ and $G_{2}$ ), the SPE of the extended game are in one-to-one correspondence with the equilibria of the $2 \times 2$ game $\widetilde{G}$

\begin{tabular}{ccc|c|} 
& \multicolumn{2}{c}{ firm 2} \\
& \multicolumn{2}{c}{$L$} & \multicolumn{1}{c}{$E$} \\
\cline { 3 - 4 } firm 1 & $E$ & $\Pi_{1}^{L}, \Pi_{2}^{F}$ & $\Pi_{1}^{N}, \Pi_{2}^{N}$ \\
\cline { 3 - 4 } & $L$ & $\Pi_{1}^{N}, \Pi_{2}^{N}$ & $\Pi_{1}^{F}, \Pi_{2}^{L}$ \\
\cline { 3 - 4 } & & &
\end{tabular}

where E and L stand for the actions "move early" and "move late", respectively.

We know that the following relations hold (see Propositions 1-3 and their proofs for justifications): $\Pi_{1}^{L} \geq \Pi_{1}^{N}, \Pi_{1}^{F} \geq \Pi_{1}^{N}, \Pi_{2}^{L} \geq \Pi_{2}^{N}, \Pi_{2}^{F} \geq \Pi_{2}^{N}$. Hence, there are two purestrategy Nash equilibria in the extended game: $(E, L)$ with payoffs $\left(\Pi_{1}^{L}, \Pi_{2}^{F}\right)$ and $(L, E)$ with payoffs $\left(\Pi_{1}^{F}, \Pi_{2}^{L}\right)$. This is a standard coordination game with two pure-strategy equilibria and one mixed-strategy equilibrium. We now consider the pure-strategy equilibrium selection problem according to two well-known criteria: Pareto dominance and risk dominance. ${ }^{21}$ Equilibrium $(E, L)$ risk-dominates equilibrium $(L, E)$ if

$$
\left(\Pi_{1}^{L}-\Pi_{1}^{N}\right)\left(\Pi_{2}^{F}-\Pi_{2}^{N}\right)>\left(\Pi_{1}^{F}-\Pi_{1}^{N}\right)\left(\Pi_{2}^{L}-\Pi_{2}^{N}\right) .
$$

That is, one equilibrium risk-dominates the other if the former is associated with the larger product of deviation losses. We now provide an argument explaining the concept of riskdominance, along with some clarifying intuition.

Denote by $p$ and $q$ the probabilities with which firms 1 and 2 select action $E$, respectively, under mixed-strategy play. Game $\widetilde{G}$ has a unique mixed equilibrium given by

$$
p^{*}=\frac{\Pi_{2}^{L}-\Pi_{2}^{N}}{\left(\Pi_{2}^{L}-\Pi_{2}^{N}\right)+\left(\Pi_{2}^{F}-\Pi_{2}^{N}\right)} \text { and } q^{*}=\frac{\Pi_{1}^{L}-\Pi_{1}^{N}}{\left(\Pi_{1}^{L}-\Pi_{1}^{N}\right)+\left(\Pi_{1}^{F}-\Pi_{1}^{N}\right)} .
$$

${ }^{20} \mathrm{~A}$ more compelling interpretation of this general scheme in the present setting is as follows: Firms decide at the beginning of Period 1 whether they enter the market or not in that period, and then choose their price in the second period, upon observing the number of firms that have entered.

${ }^{21}$ In view of the strictness of the two pure-strategy equilibrium points here, none of the standard refinements of Nash equilibrium for normal-form games (perfection, properness or strategic stability, see e.g. Fudenberg and Tirole (1991) for definitions) can be invoked to rule out one of the equilibrium points. Thus, riskdominance has a rather distinct scope of application relative to the above refinement concepts. 
Of the three equilibria, $(E, L)$ and $(L, E)$ are stable while $\left(p^{*}, q^{*}\right)$ is unstable, for a broad class of dynamics. It is easily shown that (6) says that a pure equilibrium risk dominates the other if the former has a larger basin of attraction in $(p, q)$ space. $^{22}$

From Lemma 5, if $a \in\left[M_{1}, M_{2}\right]$, firm 1 (high-cost) has a second-mover advantage and firm 2 (low cost) a first-mover advantage, so $(L, E)$ Pareto dominates $(E, L)$. If $a \in\left[M_{2}, \infty\right)$, both firms have a second-mover advantage, so no equilibrium is Pareto dominant.

Proposition 6 Assume linear demand and cost functions and Assumption (B). Then

(a) $(L, E)$ risk dominates $(E, L)$ for all a.

(b) $(L, E)$ Pareto dominates $(E, L)$ if a $\in\left[M_{1}, M_{2}\right]$ while the two are not comparable if $a \in\left[M_{2}, \infty\right)$. (L,E) leads to higher profits for the leader if a $\in\left[M_{2}, M_{3}\right)$ and higher profits for the follower if $a \in\left[M_{3}, \infty\right)$, where $M_{3}$ is the value of a for which $\Pi_{2}^{L}=\Pi_{1}^{F}$, or

$$
M_{3} \triangleq \frac{\left[4(2-b)(2+3 b)-b^{3}(10-b)+4 \sqrt{2}(1+b)\left(2-b^{2}\right)^{\frac{3}{2}}\right]\left(c_{1}-c_{2}\right)+\left(4-b-3 b^{2}\right) b^{3} c_{2}}{b^{3}(4+3 b)} .
$$

Thus, invoking the Hamilton-Slutsky timing scheme, augmented with the presumption that the firms are interested in minimizing the risk of coordination failure in their choice of timing, yields the unique outcome of sequential play with the more efficient firm as leader and the less efficient firm as follower. The reinforcement of this selection via Pareto-dominance holds only with a further restriction.

To interpret Part (b), it is instructive to rephrase it equivalently in terms of unit costs: $(L, E)$ leads to higher (lower) profits for the leader if the two units costs are sufficiently different (close). This shows that a second mover benefit still lurks in the background since the follower makes higher profits provided his cost disadvantage is not too great.

The present setting, relying on the natural endogenous timing scheme of Hamilton and Slutsky (1990) coupled with risk-dominance as a selection criterion, provides a simple and compelling explanation for the endogenous emergence of price leadership. The sequentialmove outcome creates a profit surplus relative to the simultaneous-move outcome, and riskdominance provides an interesting way of attributing roles to the firms that allocate this surplus between them, with more of this surplus going to the follower.

\footnotetext{
22In a coordination game, players always face strategic uncertainty about rivals' moves. If players are interested in minimizing the risk of coordination failure, they will tend to coordinate on the risk-dominant equilibrium, even when it is Pareto-dominated by another pure equilibrium. This explains the predictive success of risk-dominance in experimental studies, as well as in evolutionary games chracterized by experimentation and myopic learning (see e.g. Kandori et al, 1993 and Ellison, 1993).
} 
It is important to stress here that the risk dominance criterion is applied to the reduced game $\widetilde{G}$, and not to the entire two-stage extended game. The two options are a priori entirely different, as the full extended game has uncountably many strategies and lots of Nash equilibria that are not subgame-perfect. It is not clear whether the concept of risk dominance is meaningful for such a game, and if so, how it could be characterized. In view of the uniqueness of the Nash equilibria in all the four subgames of the extended game and the plausible restriction to SPE in this setting, our use of risk dominance on the reduced game as a game per se appears rather natural. Its interpretation is that the two firms wish to minimize the risk of coordination failure in their timing choices.

This methodological simplification is in line with the related literature, as we now argue. With a similar motivation, van Damme and Hurkens (1998) consider endogenous timing for the same price game, but according to Hamilton and Slutsky's extended game with action commitment. Due to the different endogenous timing scheme used, their extended game is different from ours, and on a priori grounds cannot be expected to yield the same result (indeed, see our Footnote 9 to this effect.) They also replace the second stage of the extended game by its unique equilibrium payoff and use risk-dominance on the resulting reduced game, just as we do here. Their analysis is more complicated as their extended game does not reduce, as ours does, to a $2 \times 2$ matrix game, but rather to a game with a continuum of actions. As a result, their analysis requires the full tracing procedure ${ }^{23}$.

Given that their analysis and ours select the same outcome, the two results reinforce each other in establishing the robustness of the same intuitive result with respect to the timing scheme invoked. Furthermore, this outcome is also confirmed by other related papers that produce endogenous sequencing of moves with the efficient firm as leader for duopolies in different frameworks: Van Damme and Hurkens (1999) for standard Cournot competition, Deneckere and Kovenock (1992) for Bertrand competition with homogeneous goods and capacity constraints, and Cabrales, Garcia-Fontes and Motta (2000) for Bertrand competition with vertically differentiated goods ${ }^{24}$.

23They also apply risk dominance to a $2 \times 2$ game, obtained by restricting each player to use only the equilibrium actions from the extended game. The resulting simplified analysis leads to a reversal of their result, in that the endogenous leader would then be the high-cost firm!

24 This paper presents an experimental study of the standard two-stage vertical differentiation game, wherein 


\section{Proofs}

This section provides the proofs for all the results of this paper. All the lattice-theoretic concepts and results used here are summarized in simple form in the appendix.

\section{Proof of Proposition 1.}

Since $\log \Pi_{i}\left(p_{1}, p_{2}\right)=\log \left(p_{i}-c_{i}\right)+\log D_{i}\left(p_{1}, p_{2}\right)$ it follows from Assumption (A2) that $\log \Pi_{i}$ is strictly supermodular in $\left(p_{1}, p_{2}\right)$. Applying Topkis's Theorem to this transformed payoff function, we get that every selection from each firm's reaction correspondence is a nondecreasing function of the rival's price. Hence, the Bertrand duopoly is a supermodular game here, and existence of a pure-strategy Nash equilibrium follows from Tarski's Theorem (see Appendix). Existence of a SPE for each of the games $G_{1}$ and $G_{2}$ follows in a standard way from the continuity of the payoffs and the compactness of the action sets.

As shown in [Amir, Grilo and Jin (1999), Proposition 2.4], Stackelberg prices are higher than Nash prices. This follows from the following argument, say for Game $G_{1}$. Let $\left(p_{1}^{S}, p_{2}^{S}\right)$ be the price pair associated with an arbitrary Stackelberg equilibrium of game $G_{1}$ and $\left(p_{1}^{N}, p_{2}^{N}\right)$ be the largest Bertrand equilibrium of game $G$, which is the Pareto-preferred Bertrand equilibrium [by Theorem 7 of Milgrom and Roberts, 1990]. Then

$$
\begin{aligned}
\left(p_{1}^{S}-c_{1}\right) D_{1}\left(p_{1}^{S}, p_{2}^{S}\right) & \geq\left(p_{1}^{N}-c_{1}\right) D_{1}\left(p_{1}^{N}, p_{2}^{N}\right) \\
& \geq\left(p_{1}^{S}-c_{1}\right) D_{1}\left(p_{1}^{S}, p_{2}^{N}\right)
\end{aligned}
$$

where the first inequality follows from the fact that a leader's payoff in this game cannot be worse than his Nash payoff ${ }^{25}$, and the second from the Nash equilibrium property. Since $D_{1}\left(p_{1}^{S},.\right)$ is strictly increasing, (8) implies that $p_{2}^{S} \geq p_{2}^{N}$. Since both $\left(p_{1}^{S}, p_{2}^{S}\right)$ and $\left(p_{1}^{N}, p_{2}^{N}\right)$ lie on $r_{2}$ and every selection of $r_{2}$ is nondecreasing, $p_{2}^{S} \geq p_{2}^{N}$ implies that $p_{1}^{S} \geq p_{1}^{N}$.

Due to the facts that every selection from $r_{1}$ and $r_{2}$ is a nondecreasing function of the rival's price, and that Stackelberg prices are higher than Nash prices, there are only 3 possible

firms choose quality levels in the first stage, and then prices in the second stage. It is well-known that this game admits two SPEs (see e.g. Gabszewicz and Thisse, 1979). Risk dominance is applied only to the $2 \times 2$ game consisting of the two equilibrium quality choices of the two-stage game, rather than to the reduced game with quality choices in a compact real interval obtained upon replacing the second-stage price competition by its equilibrium payoff.

25A formal proof of this is given in [Amir et al (1999), Lemma 4.1]. See also our Footnote 13. 
ways to relate equilibrium prices in $G_{1}$ and $G_{2}$ : (i) $p_{2}^{L} \geq p_{2}^{F}$ and $p_{1}^{L} \geq p_{1}^{F}$, (ii) $p_{2}^{L} \geq p_{2}^{F}$ and $p_{1}^{L} \leq p_{1}^{F}$, and (iii) $p_{2}^{L} \leq p_{2}^{F}$ and $p_{1}^{L} \geq p_{1}^{F}$. These cases are depicted in Figure 1 . We now analyse each case separately:

Case (i). Consider the following inequalities relating games $G_{1}$ and $G_{2}$ :

$$
\begin{aligned}
\left(p_{1}^{F}-c_{1}\right) D_{1}\left(p_{1}^{F}, p_{2}^{L}\right) & \geq\left(p_{1}^{L}-c_{1}\right) D_{1}\left(p_{1}^{L}, p_{2}^{L}\right) \\
& \geq\left(p_{1}^{L}-c_{1}\right) D_{1}\left(p_{1}^{L}, p_{2}^{F}\right)
\end{aligned}
$$

where the first inequality for firm 1 follows from the definition of Stackelberg equilibrium, and the second from the facts that $p_{2}^{L} \geq p_{2}^{F}$ and $D_{1}\left(p_{1}^{S},.\right)$ is increasing. This says that the profit of firm 1 is higher in game $G_{2}$ than in game $G_{1}$, so that firm 1 prefers game $G_{2}$ to game $G_{1}$. A similar argument shows that firm 2 prefers game $G_{1}$ to game $G_{2}$.

Case (ii). Here, it can be seen that the above argument for Case (i) can be applied step-for-step only for firm 1, so we can conclude only that firm 1 prefers to be a follower.

Case (iii). Here, it can be seen that the argument of Case (i) can be applied only for firm 2, so we can conclude only that firm 2 prefers to be a follower.

Overall, this establishes that there is always at least one firm that prefers to follow.

\section{Proof of Proposition 2.}

Here, we know from Topkis's Theorem that all the selections from both firms' reaction correspondences are nonincreasing. Existence of equilibrium for all three games follows from the same arguments as in the proof of Proposition 1, upon using the trick that the game is supermodular (and thus has upward-sloping reaction correspondences) if one thinks of firm 1 (say) as choosing $-p_{1}$ (instead of $p_{1}$ ), see Milgrom and Roberts (1990).

[Amir, Grilo and Jin, (1999), Lemma 4.1] proves that, under either Assumption (A2) or (A3), both firms prefer their Stackelberg leader payoff to their largest Bertrand equilibrium payoff. Likewise, [Amir, Grilo and Jin (1999), Proposition 2.5] shows that both firms prefer their worst Bertrand equilibrium payoffs (the one with lowest prices, from Milgrom and Roberts, 1990) to their Stackelberg follower payoffs. Putting these two results together, it follows that both players have a first-mover advantage.

\section{Proof of Proposition 3.}


Here, existence of equilibrium for all three games follows from the assumptions (including quasi-concavity for game $G$ ) in a standard way.

We know from Topkis's Theorem that all the selections from $r_{1}$ are nonincreasing while all the selections from $r_{2}$ are nondecreasing. [Amir, Grilo and Jin (1999), Lemma 4.1] shows that firm 1 prefers its Stackelberg leader payoff to its Bertrand equilibrium payoff, i.e. game $G_{1}$ to game $G$. [Amir, Grilo and Jin (1999), Proposition 2.6] shows that firm 1 prefers its Bertrand equilibrium payoff to its Stackelberg follower payoff, i.e. game $G$ to game $G_{2}$. It follows that firm 1 prefers game $G_{1}$ to game $G_{2}$, or that it has a first-mover advantage.

\section{Proof of Lemma 4.}

From firm $i$ 's best-response problem $\max _{p_{i}}\left(p_{i}-c_{i}\right)\left(a-p_{i}+b p_{j}\right), i, j=1,2, i \neq j$, we

obtain its reaction curve $r_{i}\left(p_{j}\right)=\frac{a+b p_{j}+c_{i}}{2}, p_{j} \in P_{j}$. The pair of Bertrand-Nash equilibrium prices (3) is the unique point where the two reaction curves intersect.

To find Stackelberg point in game $G_{i}$, firm $i$ maximizes its profit along $r_{j}$, i.e. its objective function is $\Pi_{i}\left[p_{i}, r_{j}\left(p_{i}\right)\right]=\left(p_{i}-c_{i}\right)\left[a-p_{i}+b \frac{a+b p_{i}+c_{j}}{2}\right]$. This yields $p_{i}^{L}$ as given in (4a). The Stackelberg equilibrium price of the follower $p_{j}^{F}$ (see $4 \mathrm{a}$ ) follows by substituting $p_{i}^{L}$ into $r_{j}$.

To be sure that the solutions of all three games are interior, Assumption $(B)$ is easily seen to be what is needed.

\section{Proof of Lemma 5.}

Our arguments here are based on simple but tedious (closed-form) computations. Consider the indifference relations

$$
\Pi_{2}^{L}-\Pi_{2}^{F}=0
$$

and

$$
\Pi_{1}^{L}-\Pi_{1}^{F}=0
$$

where $\Pi_{2}^{L}, \Pi_{2}^{F}\left(\Pi_{1}^{L}, \Pi_{1}^{F}\right)$ are the equilibrium profits of firm 2 (firm 1$)$ in games $G_{2}, G_{1}\left(G_{1}, G_{2}\right)$, respectively. Equations (4b) provide expressions for all four profit levels. It can be easily seen that (9) is a quadratic equation with respect to the parameter $a$. Hence, the equation has two solutions, in terms of $a$. But the smaller root does not belong to the interval of feasible $a$ 's, and is therefore not considered. Only the largest root, which is given in (5) and 
denoted by $M_{2}$ is a valid solution of (9). Since (9) is a quadratic equation w.r.t. $a$ and the term $a^{2}$ has a negative coefficient, it follows that $\Pi_{2}^{L}-\Pi_{2}^{F} \geq 0$ if and only if $a \in\left[M_{1}, M_{2}\right]$.

Concerning (10), it can be easily shown using arguments analogous to the above that, given $c_{1}>c_{2}$ and our parameter restrictions $(B)$, we always have $\Pi_{1}^{L} \leq \Pi_{1}^{F}$, so that firm 1 always has a second-mover advantage here.

Now let us prove that the scope for firm 2's first-mover advantage, i.e. $M_{2}-M_{1}$, increases with the cost difference $c_{1}-c_{2}$. Direct calculations lead to

$$
\frac{\partial\left(M_{2}-M_{1}\right)}{\partial c_{1}}=-\frac{\partial\left(M_{2}-M_{1}\right)}{\partial c_{2}}=\frac{(1+b)\left(2 b^{2}-4+(2+b) \sqrt{4-2 b^{2}}\right)}{(2+b)(4+3 b)}>0
$$

\section{Proof of Proposition 6.}

(a) To prove that equilibrium $(L, E)$ risk-dominates equilibrium $(E, L)$, or that (see $(6))$

$$
\Delta \triangleq\left(\Pi_{1}^{L}-\Pi_{1}^{N}\right)\left(\Pi_{2}^{F}-\Pi_{2}^{N}\right)-\left(\Pi_{1}^{F}-\Pi_{1}^{N}\right)\left(\Pi_{2}^{L}-\Pi_{2}^{N}\right)<0
$$

substitute $(4 b)$ with proper indexes into $\Delta$. This leads to $\Delta=\frac{N}{D}$, where

$$
\begin{aligned}
N \triangleq & b^{7}(b+1)\left(c_{1}-c_{2}\right)\left[2 a+(b-1)\left(c_{1}+c_{2}\right)\right]\left[2 a^{2}\left(b^{3}-8 b-8\right)+\right. \\
& \left.+2 a\left(b^{4}-b^{3}-8 b^{2}+8\right)\left(c_{1}+c_{2}\right)+b\left(b^{4}-7 b^{2}+8\right)\left(c_{1}^{2}+c_{2}^{2}\right)-4 c_{1} c_{2}\left(b^{2}-2\right)^{2}\right],
\end{aligned}
$$

and $D \triangleq 128\left(b^{2}-4\right)^{2}\left(2-b^{2}\right)^{3}$.

It is easy to verify that $D>0$ because $0<b<1$. Let us analyse the sign of each term of $N$ using the assumptions $0<b<1$ and $c_{1}>c_{2}$. Using Assumption (B), it can be seen that $2 a+(b-1)\left(c_{1}+c_{2}\right) \geq 0$ for all feasible $a$. We now sign the term

$$
\left(2 a^{2}\left(b^{3}-8 b-8\right)+2 a\left(b^{4}-b^{3}-8 b^{2}+8\right)\left(c_{1}+c_{2}\right)+b\left(b^{4}-7 b^{2}+8\right)\left(c_{1}^{2}+c_{2}^{2}\right)-4 c_{1} c_{2}\left(b^{2}-2\right)^{2}\right)
$$

Setting it equal to zero and solving w.r.t. $a$ gives two roots. As (11) is quadratic in $a$, one can show that (11) is negative if $a$ is higher than

$$
\widehat{a}=-\frac{\left(c_{1}+c_{2}\right)\left(b^{4}-b^{3}-8 b^{2}+8\right)+(b+1)\left(c_{1}-c_{2}\right) \sqrt{\left(4-b^{2}\right)\left(b^{4}-12 b^{2}+16\right)}}{2\left(b^{3}-8 b-8\right)} .
$$

It can be shown that $\widehat{a}<M_{1}$ (cf. Assumption B) under our conditions here, so that expression (11) is negative for all feasible values of $a$. 
Summarizing all these sign results, we conclude that $\Delta=\frac{N}{D}$ is negative for all feasible $a$. Hence, equilibrium $(L, E)$ risk-dominates equilibrium $(E, L)$.

(b) The first part follows directly from Lemma 5. Indeed, we have already shown that equilibrium $(L, E)$ Pareto-dominates equilibrium $(E, L)$ when $a \in\left[M_{1}, M_{2}\right]$, i.e. $\Pi_{1}^{F}>\Pi_{1}^{L}$ and $\Pi_{2}^{L}>\Pi_{2}^{F}$. And when $a \in\left[M_{2}, \infty\right)$, both firms have a second-mover advantage, i.e. $\Pi_{1}^{F}>\Pi_{1}^{L}$ and $\Pi_{2}^{F}>\Pi_{2}^{L}$. Hence, neither of the equilibria Pareto-dominates the other.

As for the profit comparison, it follows from a tedious computation using (4b) that $\Pi_{2}^{L}>($ resp. $<) \Pi_{1}^{F}$ if $a \in\left[M_{1}, M_{3}\right]$ (resp. $a \in\left[M_{3}, \infty\right)$.) The details are left out.

\section{Appendix}

Here, we provide a brief but self-contained summary of all the lattice-theoretic notions and results used in the present paper, in the simple framework of real action and parameter spaces: Every result presented here is a special case of the indicated original result.

A function $F:[0, \infty)^{2} \rightarrow \mathbb{R}$ is strictly supermodular (submodular) if

$$
F\left(x_{1}, y_{1}\right)-F\left(x_{2}, y_{1}\right)>(<) F\left(x_{1}, y_{2}\right)-F\left(x_{2}, y_{2}\right) \text { for all } x_{1}>x_{2}, y_{1}>y_{2} .
$$

$F$ is strictly $\log$-supermodular if $\log F$ is strictly supermodular.

Supermodularity and submodularity have complete characterizations in terms of the sign of cross-partial derivatives in case of smooth objective functions (Topkis, 1978). On the other hand, the strict versions of these notions can be given separate (related) necessary and sufficient conditions. Let $F$ be twice continuously differentiable. If $\frac{\partial^{2} F}{\partial x \partial y}>(<) 0, \forall x, y$, then $F$ is strictly supermodular (strictly submodular). Conversely, if $F$ is strictly supermodular

(strictly submodular), then we have $\frac{\partial^{2} F}{\partial x \partial y} \geq(\leq) 0, \forall x, y$. The latter inequality is equivalent to supermodularity (submodularity) of $F$ defined by (A.1) with a nonstrict inequality.

The monotonicity theorem repeatedly used in this paper is due to Topkis (1978).

Theorem A.1. Every function $x^{*}(y) \in \operatorname{argmax}_{x \geq 0} F(x, y)$ is nondecreasing (nonincreasing) in $y$ if $F$ is strictly supermodular (strictly submodular) in $(x, y)$.

In this paper, Topkis's Theorem is applied to the log of each firm's profit function.

A normal-form game with compact real action sets is supermodular (log-supermodular) if the payoffs are strictly supermodular (log-supermodular). In both cases, we say the game 
has strict strategic complementarities (in that optimal reactions are all nondecreasing.) A two-player game with strict submodular (log-submodular) payoffs becomes a supermodular (log-supermodular) game once we reverse the order on one player's action set.

We close with a statement of the associated fixed-point theorem of Tarski (1955), which establishes that supermodular games have pure-strategy equilibria, since every selection from their best response correspondence is nondecreasing, by Theorem A.1.

Theorem A.2 Let $K_{1}, K_{2}$ be compact intervals and $f: K_{1} \times K_{2} \rightarrow K_{1} \times K_{2}$ be nondecreasing. The set of fixed-points of $f$ is nonempty and contains smallest and largest elements.

\section{REFERENCES}

Amir, R. and I. Grilo (1999). Stackelberg versus Cournot Equilibrium. Games and Economic Behavior, 26, 1-21.

Amir, R. (1995). Endogenous Timing in Two-Player Games: A Counterexample. Games and Economic Behaviour, 9, 234-237.

Amir, R., I. Grilo and J. Jin (1999). Demand-Induced Endogenous Price Leadership, International Game Theory Review, 1, 219-240.

Anderson, S. and M. Engers (1992). Stackelberg vs. Cournot Oligopoly Equilibrium. International Journal of Industrial Organization, 10, 127-135.

d'Aspremont, C. and L.-A. Gerard-Varet (1980). Stackelberg Solvable Games and Preplay Communication, Journal of Economic Theory, 23, 201-217.

Boyer, M. and M. Moreaux (1987). Being a Leader or a Follower: Reflections on the Distribution of Roles in Duopoly. International Journal of Industrial Organization, 5, 175-192.

Cabrales, A., W. Garcia-Fontes and M. Motta (2000), Risk Dominance Selects the Leader: An Experimental Analysis, International Journal of Industrial Organization, 18, 137-162.

Caplin, A. and B. Nalebuff (1991), Aggregation and Imperfect Competition: On the Existence of Equilibrium, Econometrica, 59, 25-69.

Cooper, T. (1986), Most-Favored Pricing Policy and Tacit Collusion, Rand Journal of Economics, 17, 377-388.

Cooper, R., D. DeJong, R. Forsythe, and T. Ross (1990). Selection Criteria in Coordination Games: Some Experimental Results. American Economic Review, 80, 218-33. 
van Damme, E. and S. Hurkens (1996). Commitment-Robust Equilibria and Endogenous Timing. Games and Economic Behavior; 15, 290-311.

van Damme, E. and S. Hurkens (1998), Endogenous Price Leadership, Economics W. P. 289, University of Pompeu Fabra.

van Damme, E. and S. Hurkens (1999). Endogenous Stackelberg Leadership. Games and Economic Behavior; 28, 105-129.

Daughety, A (1990), Beneficial Concentration,American Economic Review 80, 1231-37.

Daughety, A. and J. Reinganum (1994). Asymmetric Information Acquisition and Behavior in Role Choice Models: An Endogenously Generated Signaling Game. International Economic Review, 35, 795-819.

Deneckere, R. and D. Kovenock (1992). Price Leadership. Review of Economic Studies, 59, 143-162.

Dowrick, S. (1986). Von Stackelberg and Cournot Duopoly: Choosing Roles. Rand Journal of Economics, 17, 251-260.

Ellison, G. (1993), Learning, Local Interaction and Coordination, Econometrica, 61, 1047-1071.

Friedman, J. (1977). Oligopoly and the Theory of Games. New York: Cambridge University Press.

Fudenberg, D. and J. Tirole (1991), Game Theory, MIT Press, Cambridge.

Gabszewicz, J. and J. Thisse (1979), Price Competition, Quality and Income Disparities, Journal of Economic Theory, 20, 340-359.

Gal-Or, E. (1985). First Mover and Second Mover Advantages. International Economic Review, 26, 649-653.

Hamilton, J. and S. Slutsky (1990). Endogenous Timing in Duopoly Games: Stackelberg or Cournot Equilibria. Games and Economic Behaviour, 2, 29-46.

Harsanyi, J. and R. Selten (1988), A General Theory of Equilibrium Selection in Games, MIT Press, Cambridge, MA.

Kandori, M., G. Mailath, and R. Rob (1993). Learning, Mutation and Long-Run Equilibria in Games, Econometrica, 61, 29-56.

Leininger, W. (1993). More Efficient Rent-Seeking - A Munchhausen Solution, Public Choice, 75, 43-62. 
Mailath, G. (1993). Endogenous Sequencing of Firm Decisions. Journal of Economic Theory, 59, 169-182.

Markham, J. (1951). The Nature and Significance of Price Leadership. American Economic Review, 41, 891-905.

Milgrom, P. and J. Roberts (1990). Rationalizability, Learning and Equilibrium in Games with Strategic Complementarities. Econometrica, 58, 1255-1277.

Pastine, I. and T. Pastine (2000), On Endogenous Leadership in Price Competition, CEPR discussion paper \#3054.

Robson, A. (1990). Duopoly with Endogenous Strategic Timing: Stackelberg Regained. International Economic Review, 31, 263-274.

Rosenthal, R. (1991). A Note on Robustness of Equilibria with Respect to Commitment Opportunities. Games and Economic Behavior, 3, 237-43.

Salop, S.C. (1986), Practices that (Credibly) Facilitate Oligopoly Coordination, in J. Stiglitz and G. Mathewson, eds., New Developments in the Analysis of Market Structure, Cambridge: MIT Press.

von Stackelberg, H. (1934). Marktform und Gleichgewicht. Springer, Vienna.

Stigler, G. (1947). The Kinked Oligopoly Demand Curve and Rigid Prices. Journal of Political Economy, 55, 432-449.

Tarski, A. (1955). A lattice-Theoretical Fixed-Point Theorem and its Applications. Pacific Journal of Mathematics, 5, 285-309.

Topkis, D. (1978). Minimizing a Submodular Function on a Lattice. Operations Research, 26, 305-321.

Van Huyck, J., Battalio, R. and Beil, R. (1990). Tacit Coordination Games, Strategic Uncertainty, and Coordination Failure, American Economic Review, 80, 234-48.

Vives, X. (1990). Nash Equilibrium with Strategic Complementarities. Journal of Mathematical Economics, 19, 305-321.

Vives, X. (2000). Oligopoly Pricing: Old Ideas and New Tools. MIT Press, Cambridge. 